\title{
All Tree Level Scattering Amplitudes in Chern-Simons Theories with Fundamental Matter
}

\author{
Karthik Inbasekar, ${ }^{1, *}$ Sachin Jain, ${ }^{2, \dagger}$ Pranjal Nayak, ${ }^{3, *}$ and V. Umesh ${ }^{4,}$ \\ ${ }^{1}$ Faculty of Exact Sciences, School of Physics and Astronomy, Tel Aviv University, Ramat Aviv 69978, Israel \\ ${ }^{2}$ Indian Institute of Science Education and Research, Homi Bhabha Road, Pashan, Pune 411 008, India \\ ${ }^{3}$ Department of Theoretical Physics, Tata Institute of Fundamental Research, Navy Nagar, Mumbai 400005, India \\ and Department of Physics and Astronomy, 265 Chemistry-Physics Building, University of Kentucky, \\ Lexington, Kentucky 40506, USA \\ ${ }^{4}$ National Institute for Theoretical Physics, School of Physics and Mandelstam Institute for Theoretical Physics, \\ University of the Witwatersrand, Johannesburg Wits 2050, South Africa
}

(Received 13 February 2018; published 15 October 2018)

\begin{abstract}
We show that Britto-Cachazo-Feng-Witten (BCFW) recursion relations can be used to compute all tree level scattering amplitudes in terms of $2 \rightarrow 2$ scattering amplitude in $\mathrm{U}(N) \mathcal{N}=2$ Chern-Simons (CS) theory coupled to matter in the fundamental representation. As a by-product, we also obtain a recursion relation for the CS theory coupled to regular fermions, even though in this case standard BCFW deformations do not have a good asymptotic behavior. Moreover, at large $N, 2 \rightarrow 2$ scattering can be computed exactly to all orders in 't Hooft coupling as was done in earlier works by some of the authors. In particular, for $\mathcal{N}=2$ theory, it was shown that $2 \rightarrow 2$ scattering is tree level exact to all orders except in the anyonic channel [K. Inbasekar et al., J. High Energy Phys. 10 (2015) 176], where it gets renormalized by a simple function of 't Hooft coupling. This suggests that it may be possible to compute the all loop exact result for arbitrary higher-point scattering amplitudes at large $N$.
\end{abstract}

DOI: 10.1103/PhysRevLett.121.161601

Introduction.-Chern-Simons (CS) gauge theories coupled to matter fields have a wide variety of applications in areas as diverse as quantum Hall physics, anyonic physics, topology of three manifolds, quantum gravity via the AdS/CFT correspondence, etc. In particular, CS theories coupled to matter in the fundamental representation $[1,2]$ are conjectured to enjoy a strong-weak duality, which follows from the study of their corresponding bulk duals [2-5]. Moreover, at large $N$, these theories are exactly solvable $[1,2,6,7]$. This led to impressive large $N, \kappa$ (keeping the 't Hooft coupling $\lambda=(N / \kappa)$ fixed) computations to all orders in the 't Hooft coupling in both sides of duality and hence verifying the duality quite convincingly. These computations include exact multipoint current correlators [8-14], exact partition function [2,15-23], and exact $S$ matrices [24-27] (see also [12,28-31] for further checks of duality). Recently, the duality was made more precise in [32-35] and subsequently generalized to finite $N$ in [36-42]. An example of the strong-weak duality is the duality between CS gauge theory coupled to fundamental

Published by the American Physical Society under the terms of the Creative Commons Attribution 4.0 International license. Further distribution of this work must maintain attribution to the author(s) and the published article's title, journal citation, and DOI. Funded by SCOAP ${ }^{3}$. fermions and CS gauge theory coupled to fundamental critical bosons. Other examples include self-dual theories, such as $\mathcal{N}=1, \mathcal{N}=2$ supersymmetric CS matter theories. At large $N$, it was demonstrated that the $S$ matrix for the $2 \rightarrow 2$ scattering computed exactly to all orders in the 't Hooft coupling displays an unusual modified crossing relation [24,25,27]. Moreover, for $\mathcal{N}=2$ theory, the result is tree level exact [24] except in the anyonic channel, where it gets renormalized by a simple function of the 't Hooft coupling.

A natural question to ask would be is it possible to compute arbitrary $m \rightarrow n$ scattering amplitudes at all values of the 't Hooft coupling at large $N, \kappa$ ? Given the simplicity of the results, at least in the supersymmetric case, it is also interesting to ask if the computability of scattering amplitudes extends to finite $N, \kappa$. As a first step towards these questions, we compute all tree level amplitudes for the $\mathcal{N}=2$ theory and the regular fermionic theory. We show that a $m \rightarrow n$ scattering amplitude can be computed recursively in terms of the $2 \rightarrow 2$ scattering amplitudes in these theories. Similar recursion relations in three dimensions were first developed in [43], in the context of the AharonyBergman-Jafferis-Maldacena (ABJM) theory, and subsequently applied to other theories such as 3D superYang-Mills theory in [44] and massive $3 \mathrm{D} \mathcal{N}=2$ gauge theories in [45]. Note that, the self-dual $\mathcal{N}=2$ supersymmetric theory is particularly interesting and important since 
via RG flow, we can obtain nonsupersymmetric dual pairs, such as critical bosons coupled to CS theory and regular fermions coupled to Chern-Simons theory [12,20].

Four-point scattering amplitude.-In this Letter, we compute scattering amplitudes in fermion coupled to $\mathrm{SU}(N)$ CS theory (FCS)

$$
\int d^{3} x\left[-\frac{\kappa}{4 \pi} \epsilon^{\mu \nu \rho} \operatorname{Tr}\left(A_{\mu} \partial_{\mu} A_{\rho}-\frac{2 i}{3} A_{\mu} A_{\nu} A_{\rho}\right)+\bar{\psi} i \not D \psi\right],
$$

and in $\mathcal{N}=2$ CS matter theory coupled to a chiral multiplet given by

$$
\begin{aligned}
\mathcal{S}_{\mathcal{N}=2}^{L}= & \int d^{3} x\left[-\frac{\kappa}{4 \pi} \epsilon^{\mu \nu \rho} \operatorname{Tr}\left(A_{\mu} \partial_{\mu} A_{\rho}-\frac{2 i}{3} A_{\mu} A_{\nu} A_{\rho}\right)\right. \\
& +\bar{\psi} i \not D \psi-\mathcal{D}^{\mu} \bar{\phi} \mathcal{D}_{\mu} \phi+\frac{4 \pi^{2}}{\kappa^{2}}(\bar{\phi} \phi)^{3}+\frac{4 \pi}{\kappa}(\bar{\phi} \phi)(\bar{\psi} \psi) \\
& \left.+\frac{2 \pi}{\kappa}(\bar{\psi} \phi)(\bar{\phi} \psi)\right]
\end{aligned}
$$

For our purposes, it is convenient to introduce the spinor helicity basis [46] defined by

$p_{i}^{\alpha \beta}=p_{i}^{\mu} \sigma_{\mu}^{\alpha \beta}=\lambda_{i}^{\alpha} \lambda_{i}^{\beta}, \quad\left(p_{i}+p_{j}\right)^{2}=2 p_{i} \cdot p_{j}=\left\langle\lambda_{i}^{\alpha} \lambda_{i, \alpha}\right\rangle^{2}$.

Below we use the notation $\left\langle\lambda_{i}^{\alpha} \lambda_{j, \alpha}\right\rangle=\langle i j\rangle$. For a supersymmetric amplitude, the standard procedure involves introduction of on-shell Grassman variables $\theta$ such that the supercreation and superannihilation operators are given by

$$
A_{i}=a_{i}+\theta_{i} \alpha_{i}, \quad A_{i}^{\dagger}=\theta_{i} a_{i}^{\dagger}+\alpha_{i}^{\dagger},
$$

where $\left(a_{i}^{\dagger}, a_{i}\right)$ or $\left(\alpha_{i}^{\dagger}, \alpha_{i}\right)$ create and annihilate a boson or fermion with momenta $p_{i}$, respectively. The two on-shell supercharges for $n$-point scattering amplitudes are given by

$Q=\sum_{i=1}^{n} q_{i}=\sum_{i=1}^{n} \lambda_{i} \theta_{i}, \quad \bar{Q}=\sum_{i=1}^{n} \bar{q}_{i}=\sum_{i=1}^{n} \lambda_{i} \partial_{\theta_{i}}$.

For FCS theory in (1), the tree level $2 \rightarrow 2$ scattering amplitude is given by [25]

$A_{4}^{F}=\left\langle\bar{\psi}\left(p_{1}\right) \psi\left(p_{2}\right) \bar{\psi}\left(p_{3}\right) \psi\left(p_{4}\right)\right\rangle=\frac{\langle 12\rangle\langle 24\rangle}{\langle 23\rangle} \delta\left(\sum_{i=1}^{4} p_{i}\right)$.

For $\mathcal{N}=2$ theory in (2), the tree level $2 \rightarrow 2$ superamplitude is given by

$A_{4}^{S}=\frac{\langle 12\rangle}{\langle 23\rangle} \delta\left(\sum_{i=1}^{4} p_{i}\right) Q^{2}=\frac{\langle 12\rangle}{\langle 23\rangle} \delta\left(\sum_{i=1}^{4} p_{i}\right) \sum_{1=i<j}^{4}\langle i j\rangle \theta_{i} \theta_{j}$.
Here $A_{4}^{S}$ is the superamplitude computed using the supercreation or annihilation operators defined in (4). Any component amplitude can be obtained from (7) by picking up the coefficient of products of two $\theta$ 's.

Higher-point scattering amplitude.-Britto-CachazoFeng-Witten (BCFW) recursion relations are an efficient method to compute and express arbitrary higher-point scattering amplitudes in terms of product of lower-point amplitudes. Standard procedure for BCFW involves the deformation of two external momenta of the particles by a complex parameter $z$ such that the particles continue to remain "on shell" and the total momentum conservation of the process continues to hold. In 3D, BCFW deformations are a little different than in 4D and were first discussed in [43] (we follow their notations closely). BCFW recursion relations are applicable in $3 \mathrm{D}$ provided that the higher-point amplitudes are regular functions at both $z \rightarrow \infty$ and $z \rightarrow 0$. In the following section, we study the $z \rightarrow \infty$ (and $z \rightarrow 0$ ) behavior of the amplitudes in the theories described earlier. We find it convenient to deform color contracted (we label them as " 1 " and " 2 ") external legs. In three dimensions, momentum deformation of particles 1 and 2 can be written in terms of the spinor-helicity variables as

$$
\left(\begin{array}{l}
\hat{\lambda}_{1} \\
\hat{\lambda}_{2}
\end{array}\right)=R\left(\begin{array}{l}
\lambda_{1} \\
\lambda_{2}
\end{array}\right), \quad \text { where } R=\left(\begin{array}{cc}
\frac{z+z^{-1}}{2} & -\frac{z-z^{-1}}{2 i} \\
\frac{z-z^{-1}}{2 i} & \frac{z+z^{-1}}{2}
\end{array}\right) .
$$

In the theories (1) and (2), all three-point vertices involve gauge fields and since the CS gauge field does not have an on-shell propagating degree of freedom, it follows that only even-point functions are nonzero. This also implies that the four-point functions are fundamental building blocks for higher-point functions.

Under the deformation (8), any tree level scattering amplitude for FCS theory in (1) is not well behaved at large $z$ and hence does not obey the requirements of BCFW. However, this situation is cured for the $\mathcal{N}=2$ theory defined in (2). Additionally, conservation of the supercharges in (5) require that the on-shell spinor variables $\theta$ be deformed as

$$
\left(\begin{array}{c}
\hat{\theta}_{1} \\
\hat{\theta}_{2}
\end{array}\right)=R\left(\begin{array}{c}
\theta_{1} \\
\theta_{2}
\end{array}\right)
$$

where the $R$ matrix is defined by (8).

Let us denote the $2 n$-point superamplitude as $A_{2 n}\left(\lambda_{1}, \lambda_{2}\right.$, $\left.\ldots, \lambda_{2 n}, \theta_{1}, \theta_{2}, \ldots, \theta_{2 n}\right)$ and the deformed amplitude by $A_{2 n}\left(\hat{\lambda}_{1}, \hat{\lambda}_{2}, \ldots, \lambda_{2 n}, \hat{\theta}_{1}, \hat{\theta}_{2}, \ldots, \theta_{2 n}, z\right)$. The deformed superamplitude can be explicitly written as an expansion in the $\theta$ variables as follows:

$$
\begin{aligned}
A_{2 n}(z)= & A^{0}(z)+A^{1}(z) \hat{\theta}_{1}(z)+A^{2}(z) \hat{\theta}_{2}(z) \\
& +A^{12}(z) \hat{\theta}_{1}(z) \hat{\theta}_{2}(z) \\
= & A^{0}(z)+\tilde{A}^{1}(z) \theta_{1}+\tilde{A}^{2}(z) \theta_{2}+A^{12}(z) \theta_{1} \theta_{2},
\end{aligned}
$$


where in the last line of (10) we have used (8) and the fact that $\hat{\theta}_{1}(z) \hat{\theta}_{2}(z)=\theta_{1} \theta_{2}$. We have also defined

$$
\left(\begin{array}{c}
\tilde{A}^{1}(z) \\
\tilde{A}^{2}(z)
\end{array}\right)=R^{T}\left(\begin{array}{c}
A^{1}(z) \\
A^{2}(z)
\end{array}\right)
$$

where $R^{T}$ is the transpose of the $R$ matrix defined in (8), with $R R^{T}=1$. The supermomentum conservation implies that the large $z$ behavior of the superamplitude $A_{2 n}(z)$ is identical to that of the components $A^{0}$ and $A^{12}$. Hence it is sufficient to show that either $A^{0}$ or $A^{12}$ are well behaved, since supersymmetric ward identity guarantees the required behavior for the rest of the amplitudes. It is convenient to write the fields in pairwise contractions, since they transform in the fundamental representation of the gauge group. For instance, we are interested in the large $z$ behavior of amplitudes such as $\left(\bar{\psi}_{1}^{i} \phi_{2 i}\right)\left(\bar{\phi}_{3}^{j} \psi_{4 j}\right) \ldots$ and $\left(\bar{\phi}_{1}^{i} \psi_{2 i}\right)\left(\bar{\psi}_{3}^{j} \phi_{4 j}\right) \ldots$, where the ellipses represent color contracted bosonic or fermionic particles allowed by interactions in (2). These amplitudes appear in $A^{0}$ and $A^{12}$ in (10), respectively.

We have checked explicitly by Feynman diagrams that the amplitude $A^{0}=A_{6}\left(\bar{\psi}_{1} \phi_{2} \bar{\phi}_{3} \psi_{4} \bar{\phi}_{5} \phi_{6}\right)$ is well behaved. We discuss the large $z$ behavior of the general $2 n$-point amplitude using the background field method [47] in the next section.

Asymptotic behavior of amplitudes.-To understand the large $z$ behavior of various scattering amplitudes, it is extremely useful to think from the background field method point of view introduced in [47]. Here $z$ deformed particles are considered as hard particles propagating in a background of soft particles. The amplitude is modified due to the (a) modified propagator of intermediate hard particle, (b) modified contribution of various vertices, and (c) modified fermion wave function, in case an external deformed particle is a fermion. Detailed analysis shows (we follow closely $[43,47])$ that the nontrivial $z \rightarrow \infty$ behavior of the amplitude is due to diagrams of the kind depicted in Fig. 1. The values of these diagrams are
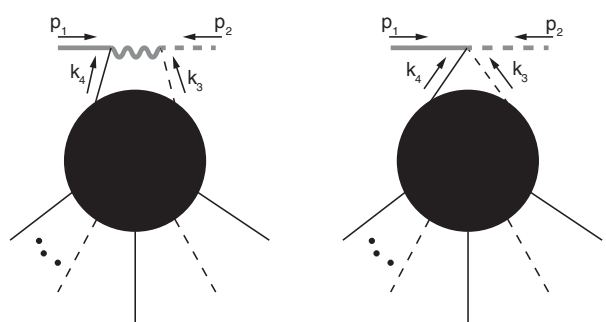

FIG. 1. The diagrams that have a nonregular $z \rightarrow \infty$ behavior. $\mathcal{O}(z)$ part of these two diagrams cancel against each other to give a regular $z \rightarrow \infty$ behavior of the total amplitude. In the above diagram, the solid lines correspond to fermions and the dashed lines correspond to bosons. This amplitude appears in $A^{0}$ in (10). The gray colored lines correspond to deformed hard particle.

gauge-field exchange: $\frac{4 \pi i}{\kappa}\left\langle k_{4}\left|\gamma^{\mu}\right| 1\right\rangle \frac{k_{3}^{\nu} p_{2}^{\rho}}{\left(k_{3}+p_{2}\right)^{2}} \epsilon_{\mu \nu \rho}$

$$
\text { contact vertex: }-\frac{2 \pi}{\kappa}\left\langle k_{4} \mid 1\right\rangle \text {. }
$$

Under the $1-2 z$ deformations (8), in the $z \rightarrow \infty$ limit, the $\mathcal{O}(z)$ part of the amplitude cancels and the amplitude behaves as $\mathcal{O}(1 / z)$. Hence, this amplitude has a regular $z \rightarrow \infty$ behavior for $\mathcal{N}=2$ theory. This cancellation works even for the four-point function $\left\langle\bar{\psi}_{1} \phi_{2} \bar{\phi}_{3} \psi_{4}\right\rangle$, which receives contributions from the diagrams in Fig. 1 with the blob removed and $k_{3} \rightarrow p_{3}, k_{4} \rightarrow p_{4}$ are taken to be onshell momenta. It is important to emphasize that we need minimum $\mathcal{N}=2$ amount of supersymmetry for this to work [48].

Recursion relations in $\mathcal{N}=2$ theory.-In the last section, we have demonstrated that $A^{0}$ is well behaved in large $z$. Hence, we can apply the BCFW recursion relation directly to the superamplitude in the left-hand side of (10). The recursion formula for a $2 n$-point superamplitude can be expressed in terms of lower-point superamplitudes as follows (see Fig. 2):

$$
\begin{gathered}
A_{2 n}(z=1)=\sum_{f} \int \frac{d \theta}{p_{f}^{2}}\left(z_{a ; f} \frac{z_{b ; f}^{2}-1}{z_{a ; f}^{2}-z_{b ; f}^{2}} A_{L}\left(z_{a ; f}, \theta\right) A_{R}\left(z_{a ; f}, i \theta\right)+\left(z_{a ; f} \leftrightarrow z_{b ; f}\right)\right), \\
\left(z_{a ; f}^{2}, z_{b ; f}^{2}\right)=\frac{-\left(p_{f}-p_{2}\right) \cdot\left(p_{f}+p_{1}\right) \pm \sqrt{\left(p_{f}-p_{2}\right)^{2}\left(p_{f}+p_{1}\right)^{2}}}{4 q \cdot\left(p_{f}-p_{2}\right)}
\end{gathered}
$$

where the integration is over the intermediate Grassmann variable $\theta$ and $A_{2 n}(z=1)$ is the undeformed $2 n$-point amplitude. In the above, $p_{f}$ is the undeformed momentum that runs in the factorization channel $f$ and the summation in (14) runs over all the factorization channels corresponding to different intermediate particles going on shell. Here, $z_{a ; f}$ and $z_{b ; f}$ are given by (15), where the null momenta $q$ are defined in terms of the spinor-helicity variables as 


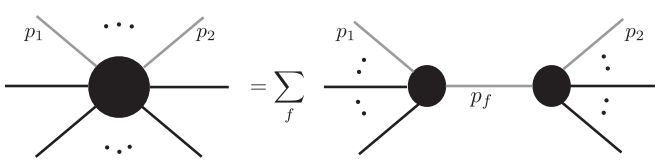

FIG. 2. Recursion formula for a $2 n$-point amplitude. The black lines denote the undeformed legs, the external gray lines represent the deformed legs, and $p_{f}$ represents the momentum in the factorization channel.

$$
q^{\alpha \beta}=\frac{1}{4}\left(\lambda_{2}+i \lambda_{1}\right)^{\alpha}\left(\lambda_{2}+i \lambda_{1}\right)^{\beta} .
$$

Note that (14) has a very similar form (but not quite the same as discussed below) to the one obtained in [43] for the ABJM theory [49] that enjoys $\mathcal{N}=6$ supersymmetry. It is remarkable that such recursion formulas exist in a theory with much lesser supersymmetry such as the one in discussion.

The appearance of square roots in expression (15) could be seen as a concern for giving rise to branch cuts in the amplitudes. However, note that $A_{L}(-z) A_{R}(-z)=$ $-A_{L}(z) A_{R}(z)$ in the integrand of (14) [50]. Also the prefactor is an odd function of $z$. Consequently the total integrand is an even function of $z_{a ; f}$ and $z_{b ; f}$ and hence only depends on $z_{a ; f}^{2}$ and $z_{b ; f}^{2}$. Moreover, the integrand is also symmetric under $z_{a ; f} \leftrightarrow z_{b ; f}$. This implies that the amplitude is only a function of $z_{a ; f}^{2}+z_{b ; f}^{2}$ and $z_{a ; f}^{2} z_{b ; f}^{2}$, and hence there are no square roots in the final expression.

As an explicit demonstration of recursion relations in (14), consider the six-point [51] amplitude $A_{6}\left(\lambda_{1} \ldots \lambda_{6}\right) \equiv$ $(\bar{\phi} \psi)(\bar{\psi} \phi)(\bar{\phi} \phi)$ in the $\mathcal{N}=2$ Superconformal ChernSimons theory. This amplitude factorizes into two channels as shown in Fig. 3.

The recursion formula can be explicitly written as

$$
\begin{aligned}
& \left\langle\bar{\phi}_{1} \psi_{2} \bar{\psi}_{3} \phi_{4} \bar{\phi}_{5} \phi_{6}\right\rangle \\
& =\left(z_{a ; f} \frac{z_{b ; f}^{2}-1}{z_{a ; f}^{2}-z_{b ; f}^{2}}\left\langle\hat{\bar{\phi}}_{1} \hat{\phi}_{f} \bar{\phi}_{5} \phi_{6}\right\rangle_{z_{a ; f}}\left\langle\hat{\bar{\phi}}_{(-f)} \hat{\psi}_{2} \bar{\psi}_{3} \phi_{4}\right\rangle_{z_{a ; f}}\right. \\
& \left.\quad+\left(z_{a ; f} \leftrightarrow z_{b ; f}\right)\right)\left.\frac{i}{p_{f}^{2}}\right|_{p_{f}=p_{234}} \\
& \quad+\left(z_{a ; f} \frac{z_{b ; f}^{2}-1}{z_{a ; f}^{2}-z_{b ; f}^{2}}\left\langle\hat{\bar{\phi}}_{1} \hat{\psi}_{f} \bar{\psi}_{3} \phi_{4}\right\rangle_{z_{a ; f}}\left\langle\hat{\bar{\psi}}_{(-f)} \hat{\psi}_{2} \bar{\phi}_{5} \phi_{6}\right\rangle_{z_{a ; f}}\right. \\
& \left.\quad+\left(z_{a ; f} \leftrightarrow z_{b ; f}\right)\right)\left.\frac{i}{p_{f}^{2}}\right|_{p_{f}=p_{256}}
\end{aligned}
$$

$$
\begin{aligned}
= & \left(\frac{32 \pi^{2} i}{\kappa^{2}}\right)\left(\frac{\left\langle 2\left|p_{4}\right| 3\right\rangle p_{12} \cdot p_{56}-\left\langle 2\left|p_{1}\right| 3\right\rangle p_{34} \cdot p_{56}}{p_{256}^{2} p_{124}^{2}}\right. \\
& +\left[\left\langle 3\left|p_{12}\right| 5\right\rangle\left(\left\langle 2\left|p_{1}\right| 5\right\rangle p_{34} \cdot p_{56}-\left\langle 2\left|p_{6}\right| 5\right\rangle p_{34} \cdot p_{12}\right)\right. \\
& \left.-\langle 34\rangle\langle 12\rangle\left(\left\langle 1\left|p_{6}\right| 4\right\rangle p_{12} \cdot p_{56}-\left\langle 1\left|p_{2}\right| 5\right\rangle\left\langle 4\left|p_{6}\right| 5\right\rangle\right)\right] \\
& \left.\times \frac{1}{p_{234}^{2} p_{123}^{2} p_{126}^{2}}\right) .
\end{aligned}
$$

Fields with hats correspond to deformed momenta. We have checked (17) explicitly by computing the relevant Feynman diagrams. It is a curious fact that the total number of Feynman graphs that contribute to $A_{6}$ is 15 . Of these, 11 are reproduced by the channel $p_{f}=p_{234}$ and the remaining four in the channel $p_{f}=p_{256}$. Moreover, we have also reproduced the correct additional poles in the respective channels. The final answer is manifestly free of any spurious poles and square roots as we argued above.

Recursion relations in the fermionic theory.-In this section, we show that the BCFW recursion relations can be used to compute $2 n$-point amplitude $A_{2 n}=\left(\bar{\psi}_{1} \psi_{2}\right) \ldots$ $\left(\bar{\psi}_{2 n-1} \psi_{2 n}\right)$ for the regular fermionic theory coupled to CS gauge field (1). If we apply (8) to this amplitude, it is easy to show that it does not have a good large $z$ (as well as $z \rightarrow 0$ ) behavior; hence we cannot readily apply the BCFW recursion relation [52] to determine all higher-point fermionic amplitudes. However, we show below that we can use the recursion relation of the $\mathcal{N}=2$ to write a recursion relation for the fermionic theory.

As a first step towards this, let us note that the Feynman diagrams for any tree level all-fermion scattering amplitude in the $\mathcal{N}=2$ theory (2) is identical to that of the tree level scattering amplitude in the fermionic theory (1). In the previous section, we proved for the $\mathcal{N}=2$ theory that an arbitrary higher-point superamplitude can be written only in terms of the four-point superamplitude. The same can be said for the component amplitudes, including the purely fermionic component amplitude [53]. Let us note that, for the four-point superamplitude, supersymmetry relates all the component four-point amplitudes to one component amplitude, which can be taken to be four-fermion scattering amplitude [see (7)]. Thus, an arbitrary higher-point component amplitude can be written only in terms of four-fermion amplitude. This can be recursively done for an arbitrary $2 n$-point amplitude; however, for simplicity, we write the recursion relation for the six-point amplitude below,

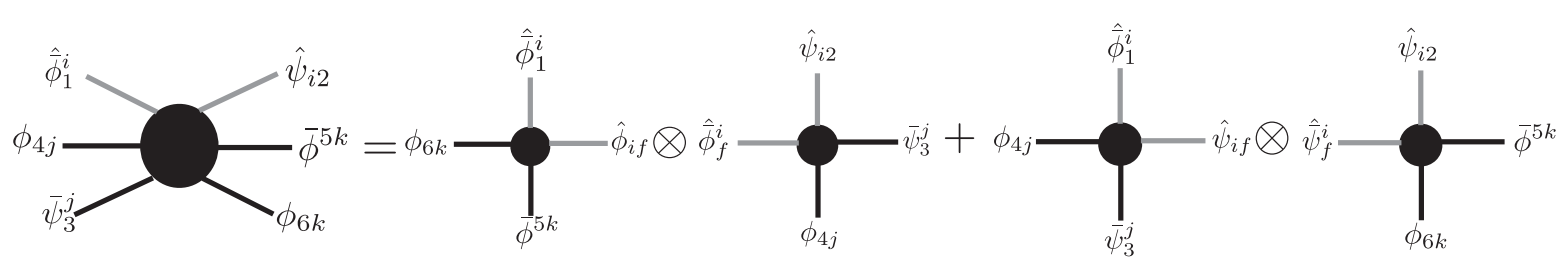

FIG. 3. BCFW recursion for the six-point amplitude. Factorization into two channels. Each four-point amplitude on the rhs is on shell. Two adjacent lines with the same color are color contracted. Note that the gray lines in particular represent the BCFW deformed legs. 


$$
\begin{aligned}
\left\langle\bar{\psi}_{1} \psi_{2} \bar{\psi}_{3} \psi_{4} \bar{\psi}_{5} \psi_{6}\right\rangle= & \left(z_{a ; f} \frac{z_{b ; f}^{2}-1}{z_{a ; f}^{2}-z_{b ; f}^{2}}\left[\frac{z_{a ; f}^{2}+1}{2 z_{a ; f}}+i \frac{z_{a ; f}^{2}-1}{2 z_{a ; f}} \frac{\langle\hat{1} 4\rangle}{\langle\hat{f} 4\rangle} \frac{\langle\hat{f} 6\rangle}{\langle\hat{2} 6\rangle}\right]\left\langle\hat{\bar{\psi}}_{1} \hat{\psi}_{f} \bar{\psi}_{3} \psi_{4}\right\rangle\left\langle\hat{\bar{\psi}}_{(-f)} \hat{\psi}_{2} \bar{\psi}_{5} \psi_{6}\right\rangle_{z_{a ; f}}\right. \\
+ & \left.\left(z_{a ; f} \leftrightarrow z_{b ; f}\right)\right)\left.\frac{1}{p_{f}^{2}}\right|_{p_{f}=p_{256}}+\left(z_{a ; f} \frac{z_{b ; f}^{2}-1}{z_{a ; f}^{2}-z_{b ; f}^{2}}\left[\frac{z_{a ; f}^{2}+1}{2 z_{a ; f}}+i \frac{z_{a ; f}^{2}-1}{2 z_{a ; f}} \frac{\langle\hat{1} 6\rangle}{\langle\hat{f} 6\rangle} \frac{\langle\hat{f} 4\rangle}{\langle\hat{2} 4\rangle}\right]\right. \\
& \left.\times\left\langle\hat{\bar{\psi}}_{1} \hat{\psi}_{f} \bar{\psi}_{5} \psi_{6}\right\rangle\left\langle\hat{\bar{\psi}}_{(-f)} \hat{\psi}_{2} \bar{\psi}_{3} \psi_{4}\right\rangle_{z_{a ; f}}+\left(z_{a ; f} \leftrightarrow z_{b ; f}\right)\right)\left.\frac{1}{p_{f}^{2}}\right|_{p_{f}=p_{234}}, \\
= & \left(\frac{16 \pi^{2} i}{\kappa^{2}}\right)\left(\frac{1}{p_{124}^{2} p_{125}^{2} p_{256}^{2}}-\frac{1}{p_{123}^{2} p_{126}^{2} p_{234}^{2}}\right)\left[-\left\langle 1\left|p_{34}\right| 2\right\rangle\left\langle 3\left|p_{56}\right| 4\right\rangle\left\langle 5\left|p_{12}\right| 6\right\rangle+\langle 56\rangle\langle 12\rangle\langle 34\rangle\right. \\
& \left.\times\left(\left\langle 5\left|p_{12}\right| 6\right\rangle\langle 56\rangle+\left\langle 3\left|p_{56}\right| 4\right\rangle\langle 34\rangle+\left\langle 1\left|p_{34}\right| 2\right\rangle\langle 12\rangle\right)\right] .
\end{aligned}
$$

The above answer is remarkably simple and is manifestly invariant under the permutations of particle pairs $\{12\},\{34\}$, and $\{56\}$, as expected.

Discussion.-In this Letter, we presented recursion relations for all tree level amplitudes in $\mathcal{N}=2 \mathrm{CS}$ matter theory and CS theory coupled to regular fermions. Below we discuss some interesting open questions for future research.

It was shown in [24] that the $2 \rightarrow 2$ scattering amplitude in the $\mathcal{N}=2$ theory does not get renormalized except in the anyonic channel, where it gets renormalized by a simple function of the 't Hooft coupling. A natural question is why, in the $\mathcal{N}=2$ theory, the scattering amplitude has such a simple form, whereas the corresponding amplitudes in the fermionic [25] and other less supersymmetric $\mathcal{N}=1$ [24] theories are quite complicated, and if the simplicity of the amplitudes continues to persist with higher-point amplitudes. It is also interesting to explore an analog of the Aharonov-Bohm phase for higher-point amplitudes. It may very well turn out that the Aharonov-Bohm phases of higher-point amplitudes are products of the AharonovBohm phases of the $2 \rightarrow 2$ amplitude. BCFW recursion relations provide a strong indication towards this result.

To answer the above questions, we need to compute higher scattering amplitudes to all orders in $\lambda$. A possible way is to investigate the Schwinger-Dyson equation. However, the Schwinger-Dyson equation approach is quite complicated even at the six-point level. A refined approach might be to look for a larger class of symmetries such as dual superconformal symmetry [54] and Yangian symmetry and use the powerful formulation of [55] to obtain results. Given the fact that these theories are exactly solvable at large $N$ as well as the fact that $\mathcal{N}=2$ theory is self-dual, it could turn out that the $\mathcal{N}=2$ theory may be one of the simplest playing grounds to develop new techniques in computing $S$ matrices to all orders [55]. Furthermore, exact solvability at large $N$ indicates that these models might even be integrable. One possible way to investigate integrability is to show the existence of an infinite-dimensional Yangian symmetry. Since these theories relate to various physical situations, any of the above exercises may provide insight into finite $N, \kappa$ computations.
We thank O. Aharony, S. Ananth, S.Banerjee, R. Gopakumar, Nima Arkani Hamed, T. Hartman, Y-t. Huang, S. Kundu, R. Loganayagam, J. Maldacena, G. Mandal, S. Minwalla, S. Mukhi, S. Raju, A. Sever, T. Sharma, R. Soni, J. Sonnenschein, S. Trivedi, and S. Wadia for helpful discussions. We would like to thank Nima Afkhami-Jeddi and Amirhossein Tajdini for collaboration during the initial stages of the project. We would like to thank Y-t. Huang for sharing a useful mathematica code with us. Special thanks S. Minwalla for very useful and critical discussions. The work of K. I. was supported in part by a center of excellence supported by the Israel Science Foundation (Grant No. 1989/14), the US-Israel binational fund (BSF) Grant No. 2012383, and the Germany Israel binational fund GIF Grant No. I-244-303.7-2013. S. J. would like to thank TIFR for hospitality at various stages of the work. Some part of the work in this Letter was completed while S. J. was a postdoctoral candidate at Cornell and his research was supported by Grant No. 488643 from the Simons Foundation. The work of P. N. is supported partly by Infosys Endowment for the study of the Quantum Structure of Space Time and Indo-Israel grant of S. Minwalla; and partly by the College of Arts and Sciences of the University of Kentucky.

*karthikin@tauex.tau.ac.il sachin.jain@iiserpune.ac.in nayak.pranjal@gmail.com §vumesh.physics@gmail.com

[1] O. Aharony, G. GurAri, and R. Yacoby, J. High Energy Phys. 03 (2012) 037.

[2] S. Giombi, S. Minwalla, S. Prakash, S. P. Trivedi, S. R. Wadia, and X. Yin, Eur. Phys. J. C 72, 2112 (2012).

[3] I. R. Klebanov and A. M. Polyakov, Phys. Lett. B 550, 213 (2002).

[4] E. Sezgin and P. Sundell, Nucl. Phys. B644, 303 (2002); B660, 403 (2003).

[5] S. Giombi and X. Yin, J. High Energy Phys. 09 (2010) 115.

[6] J. Maldacena and A. Zhiboedov, J. Phys. A 46, 214011 (2013).

[7] J. Maldacena and A. Zhiboedov, Classical Quantum Gravity 30, 104003 (2013). 
[8] O. Aharony, G. Gur-Ari, and R. Yacoby, J. High Energy Phys. 12 (2012) 028.

[9] G. Gur-Ari and R. Yacoby, J. High Energy Phys. 02 (2013) 150 .

[10] V. Gurucharan and S. Prakash, J. High Energy Phys. 09 (2014) 009.

[11] A. Bedhotiya and S. Prakash, J. High Energy Phys. 12 (2015) 032.

[12] G. GurAri and R. Yacoby, J. High Energy Phys. 11 (2015) 013.

[13] M. Geracie, M. Goykhman, and D. T. Son, J. High Energy Phys. 04 (2016) 103.

[14] G. Gur-Ari, S. A. Hartnoll, and R. Mahajan, J. High Energy Phys. 07 (2016) 090.

[15] S. Jain, S. P. Trivedi, S. R. Wadia, and S. Yokoyama, J. High Energy Phys. 10 (2012) 194.

[16] S. Yokoyama, J. High Energy Phys. 01 (2013) 052.

[17] O. Aharony, S. Giombi, G. Gur-Ari, J. Maldacena, and R. Yacoby, J. High Energy Phys. 03 (2013) 121.

[18] S. Jain, S. Minwalla, T. Sharma, T. Takimi, S. R. Wadia, and S. Yokoyama, J. High Energy Phys. 09 (2013) 009.

[19] T. Takimi, J. High Energy Phys. 07 (2013) 177.

[20] S. Jain, S. Minwalla, and S. Yokoyama, J. High Energy Phys. 11 (2013) 037.

[21] S. Yokoyama, J. High Energy Phys. 01 (2014) 148.

[22] S. Minwalla and S. Yokoyama, J. High Energy Phys. 02 (2016) 103.

[23] T. Nosaka and S. Yokoyama, J. High Energy Phys. 01 (2018) 001.

[24] K. Inbasekar, S. Jain, S. Mazumdar, S. Minwalla, V. Umesh, and S. Yokoyama, J. High Energy Phys. 10 (2015) 176.

[25] S. Jain, M. Mandlik, S. Minwalla, T. Takimi, S. R. Wadia, and S. Yokoyama, J. High Energy Phys. 04 (2015) 129.

[26] Y. Dandekar, M. Mandlik, and S. Minwalla, J. High Energy Phys. 04 (2015) 102.

[27] S. Yokoyama, J. High Energy Phys. 09 (2016) 105.

[28] S. Giombi, V. Gurucharan, V. Kirilin, S. Prakash, and E. Skvortsov, J. High Energy Phys. 01 (2017) 058.

[29] S. R. Wadia, Int. J. Mod. Phys. A 31, 1630052 (2016).

[30] D. Radicevic, D. Tong, and C. Turner, J. High Energy Phys. 12 (2016) 067.

[31] S. Giombi, arXiv:1707.06604.

[32] D. Radicevic, J. High Energy Phys. 03 (2016) 131.

[33] O. Aharony, J. High Energy Phys. 02 (2016) 093.

[34] N. Seiberg, T. Senthil, C. Wang, and E. Witten, Ann. Phys. (Amsterdam) 374, 395 (2016).

[35] A. Karch and D. Tong, Phys. Rev. X 6, 031043 (2016).

[36] A. Karch, B. Robinson, and D. Tong, J. High Energy Phys. 01 (2017) 017.

[37] P.-S. Hsin and N. Seiberg, J. High Energy Phys. 09 (2016) 095.

[38] O. Aharony, F. Benini, P.-S. Hsin, and N. Seiberg, J. High Energy Phys. 02 (2017) 072.

[39] F. Benini, P.-S. Hsin, and N. Seiberg, J. High Energy Phys. 04 (2017) 135.

[40] D. Gaiotto, Z. Komargodski, and N. Seiberg, J. High Energy Phys. 01 (2018) 110.

[41] K. Jensen and A. Karch, J. High Energy Phys. 11 (2017) 018.

[42] K. Jensen and A. Karch, J. High Energy Phys. 12 (2017) 031.

[43] D. Gang, Y.-t. Huang, E. Koh, S. Lee, and A. E. Lipstein, J. High Energy Phys. 03 (2011) 116.
[44] A. E. Lipstein and L. Mason, J. High Energy Phys. 01 (2013) 009.

[45] A. Agarwal, A. E. Lipstein, and D. Young, Phys. Rev. D 89, 045020 (2014).

[46] H. Elvang and Y.-t. Huang, Scattering Amplitudes in Gauge Theory and Gravity (Cambridge University Press, Cambridge, England, 2015).

[47] N. Arkani-Hamed and J. Kaplan, J. High Energy Phys. 04 (2008) 076.

[48] For instance, for $\mathcal{N}=1$ theory, the Lagrangian that can be found in [24] [Eq. (2.11)], (13) is modified to $(-2 \pi w / \kappa)\langle 41\rangle$, whereas (12) remains the same. Here $w$ is a free parameter in $\mathcal{N}=1$ theory. This implies that, only at $w=1$, the $N=1$ theory has a good large $z$ behavior. This is exactly the point in the $w$ line where the supersymmetry of the theory gets enhanced to $\mathcal{N}=2$.

[49] Although formula (14) looks very similar to the ABJM case, the details are different since the external matter particles are in fundamental representation. For example, in general, there will be more factorization channels here as compared to the ABJM case. For example, in the six-point function, as will be clear below, there are two factorized channels, whereas for the corresponding deformation in ABJM, there is only one factorized channel.

[50] Under $z \rightarrow-z$, both $\hat{\lambda} \rightarrow-\hat{\lambda}$ and $\hat{\eta} \rightarrow-\hat{\eta}$ [see (8) and (9)]. From (4) one can deduce that the scaling of the on-shell fields $\Phi, \bar{\Phi}$ to be $+1 /-1$ under $z \rightarrow-z$. In our BCFW deformations, we deform one $\Phi$ and one $\bar{\Phi}$, and therefore, $A_{L} A_{R} \rightarrow-A_{L} A_{R}$. See [43] for a related discussion in the context of the ABJM theory.

[51] A general six-point superamplitude in $\mathcal{N}=2$ theory can be written in terms of two independent functions as

$$
\begin{aligned}
A_{6}= & Q^{2}\left(f_{1}(p) \sum_{i=1}^{3} \epsilon^{i j k} \lambda\left(p_{j}\right) \lambda\left(p_{k}\right) \theta_{i}\right. \\
& \left.+f_{2}(p) \sum_{i=4}^{6} \epsilon^{i l m} \lambda\left(p_{l}\right) \lambda\left(p_{m}\right) \theta_{i}\right),
\end{aligned}
$$

where $Q=\sum_{i=1}^{6} \lambda_{i} \theta_{i}$ as defined in (5).

[52] There will be some nontrivial boundary terms that do not vanish and, in general, there are no good prescriptions to compute them systematically.

[53] Note that the recursion relation in the $\mathcal{N}=2$ theory (14) does not directly give $\left(\bar{\psi}_{1} \psi_{2}\right) \ldots\left(\bar{\psi}_{2 n-1} \psi_{2 n}\right)$ in terms of the lower-point fermionic amplitude. However, we can use $\mathrm{BCFW}$ relations recursively to write down any higher-point amplitude in terms of four-point amplitudes such as $(\bar{\psi} \psi)(\bar{\psi} \psi),(\bar{\phi} \phi)(\bar{\phi} \phi),(\bar{\phi} \phi)(\bar{\psi} \psi),(\bar{\phi} \psi)(\bar{\psi} \phi)$, etc. Moreover, at the level of the four-point amplitude, one can rewrite this in terms of $(\bar{\psi} \psi)(\bar{\psi} \psi)$. For example, $(\bar{\psi} \psi)(\bar{\phi} \phi)=$ $(\langle 23\rangle /\langle 24\rangle)(\bar{\psi} \psi)(\bar{\psi} \psi)$. This implies that we get a recursion relation for $\left(\bar{\psi}_{1} \psi_{2}\right) \ldots\left(\bar{\psi}_{2 n-1} \psi_{2 n}\right)$ in terms of lower-point fermionic amplitudes only. Hence this can be interpreted as a BCFW recursion relation in the regular fermionic theory coupled to CS gauge field (1).

[54] K. Inbasekar, S. Jain, S. Majumdar, P. Nayak, T. Neogi, T. Sharma, R. Sinha, and V. Umesh, arXiv:1711.02672.

[55] N. Arkani-Hamed, J. L. Bourjaily, F. Cachazo, A. B. Goncharov, A. Postnikov, and J. Trnka, Grassmannian Geometry of Scattering Amplitudes (Cambridge University Press, Cambridge, England, 2016). 